\title{
Concise Commentary: Prebiotic, Probiotic, Whatever-It's All Good for IBS-C
}

\author{
Giuseppe Chiarioni $^{1} \cdot$ Eugenio Marconato $^{1}$
}

Published online: 7 August 2019

๑) Springer Science+Business Media, LLC, part of Springer Nature 2019

\section{Introduction}

Irritable bowel syndrome (IBS) is a prevalent functional gastrointestinal disorder characterized by abdominal pain and altered bowel habits $[1,2]$. The etiology of IBS, which is currently unclear, is likely multifactorial $[1,2]$. Nevertheless, alterations of the intestinal microbiota associated with disordered local immunity, low-grade inflammation, visceral hypersensitivity, and increased permeability of the brain-gut axis have all been implicated [1]. Although alteration of the microbiota is associated with improved IBS symptoms in most studies, the mechanism of action is unclear [1, 3]. According to the World Health Organization, probiotics are living bacteria that confer health benefits when given in adequate amounts $[1,3]$. On the contrary, prebiotics are nonviable dietary substances such as non-digestible polysaccharides that serve as microbial nutrients [1,3]. A combination of prebiotics and probiotics (so-called symbiotic mixture) seems to be a worthwhile add-on to benefit IBS symptoms [1]. In this issue of Digestive Diseases and Sciences, Bahrudin and coworkers report the results of a randomized oneweek controlled trial conducted in 163 IBS patients with prevalent constipation (IBS-C) in order to investigate the efficacy of a sterilized probiotic drink containing Lactobacillus helveticus (with or without $5.85 \mathrm{~g}$ polydextrose added as a prebiotic) on gastrointestinal symptoms and intestinal physiology [3]. IBS-C patients were of Asian heritage, mostly female (78.6\%), and diagnosed according to Rome III criteria [4]. Physiologic measurements such as transit time measured with carmine red, fecal weight, and pH testing, and clinical evaluation using the Garrigues constipation questionnaire on related symptoms were conducted pre- and post-intervention. Intestinal transit time was shortened in both groups compared to baseline, whereas the fecal weight and $\mathrm{pH}$ were variable. Constipation-related symptoms were again equally improved compared with baseline by both probiotic and symbiotic drinks. The weaknesses of the study were the short treatment interval and the lack of a placebo group; strengths included a large sample size, a randomized, prospective design, the use of standardized criteria to diagnose IBS, and the objective evaluation of intestinal physiology and symptoms. In conclusion, probiotics and prebiotics benefit constipation symptoms in IBS-C associated with shortening intestinal transit time. The mechanism of action of probiotics in IBS is likely complex and deserves additional evaluation, but feeding beneficial bacteria does not appear to be the way to improve IBS treatment outcome.

\section{References}

1. Gracie DJ, Ford AC. Symbiotics in the irritable bowel syndromebetter than probiotics alone? Curr Opin Clin Nutr Metab Care. 2015;18:485-489.

2. Longstreth GF, Thompson WG, Chey WD, Houghton LA, Mearin F, Spiller RC. Functional bowel disorders. Gastroenterology. 2006;130:1480-1491.

3. Staley C, Kaiser T, Khoruts A. Clinician guide to microbiome testing. Dig Dis Sci. 2018;63:3167-3177. https://doi.org/10.1007/ s10620-018-5299-6.

4. Bahrudin MF, Rani RA, Tamil AM, Mokhtar MN, RAR Ali. Effectiveness of sterilized symbiotic drink containing Lactobacillus helveticus is comparable to probiotic alone in patients with constipation-predominant irritable bowel syndrome. Dig Dis Sci. (Epub ahead of print). https://doi.org/10.1007/s10620-019-05695 -3 .

Publisher's Note Springer Nature remains neutral with regard to jurisdictional claims in published maps and institutional affiliations.
Giuseppe Chiarioni

chiarioni@alice.it

1 Division of Gastroenterology, University of Verona, AOUI Verona, Verona, Italy 